\title{
Sub-metric spatial resolution over an extended range using differential time-domain Brillouin sensing
}

\author{
Alejandro Dominguez-Lopez*a,b, Marcelo A. Soto ${ }^{\mathrm{a}}$, Sonia Martin-Lopez ${ }^{\mathrm{b}}$, Miguel Gonzalez- \\ Herrae $^{\mathrm{b}}$ and Luc Thevenaz ${ }^{\mathrm{a}}$ \\ ${ }^{a}$ Inst. of Electrical Eng., Swiss Federal Inst. of Tech. (EPFL), CH-1015 Lausanne, Switzerland; \\ ${ }^{\mathrm{b}}$ Dept. of Electronics, University of Alcala, Polytechnic School, 28805 Alcala de Henares, Spain;
}

\begin{abstract}
This paper reports on a distributed fiber sensing scheme to achieve sub-metric spatial resolution and long sensing ranges based on a differential pulse-width pair (DPP) Brillouin sensor. The scheme uses a scanning method in which the spectral separation between the two probe sidebands is kept constant, while the optical frequency of the pump is swept to scan the Brillouin spectral response. Experimental results show that the method avoids detrimental temporal distortions of the pulses, which in a standard implementation prevent the DPP method to operate over long distances. Here we demonstrate that this novel scanning method enables distributed sensing over $37.5 \mathrm{~km}$ with a spatial resolution of $20 \mathrm{~cm}$ and a frequency uncertainty of $1.9 \mathrm{MHz}$, obtained with $2 \mathrm{k}$ averages in a measurement time of a few minutes.
\end{abstract}

Keywords: Brillouin distributed sensors, Brillouin scattering, nonlinear optics, fiber optic sensors.

\section{INTRODUCTION}

Distributed optical fiber sensing based on Brillouin optical time domain analysis ${ }^{1,2}$ (BOTDA) offers interesting capabilities to measure a distributed strain/temperature profile along optical fibers of several tens of kilometers. The interaction of a pulsed pump and a continuous-wave $(\mathrm{CW})$ probe signal allows measuring the temperature- and straindependent Brillouin gain response of the sensing fiber, with a spatial resolution given by the pump pulse width ${ }^{1,2}$. The limited response time ( $\sim 10 \mathrm{~ns})$ of the acoustic wave generated at each fiber location imposes a fundamental limitation to the spatial resolution of BOTDA sensors, which cannot reliably provide information with spatial variations below $1 \mathrm{~m}$. To achieve sub-metric spatial resolution, different approaches have been proposed based on frequency, correlation ${ }^{3,4}$ or time $^{5}$ domain. Frequency- and correlation-domain approaches allow very sharp spatial resolutions (in the order of $\mathrm{mm}$ or a few $\mathrm{cm}$ ), however the measurement range is typically limited below a few $\mathrm{km}$. On the other hand, time-domain approaches typically make use of differential pulse measurements, where the spatial resolution highly depends on the rising/falling time of the used optical pulses, thus being typically limited to tens of $\mathrm{cm}$ along mid-range sensing distances (usually $<10 \mathrm{~km}$ ). Using sophisticated modulations of the optical signals, the trade-off between spatial resolution and measurement range has been overcome for correlation- and time-domain Brillouin sensors. In the first case, the use of correlation-domain combined with phase-modulated signals and time-domain acquisition has led to measurements with a spatial resolution of $8.3 \mathrm{~mm}$ over a distance of $17.5 \mathrm{~km}$, representing more than 2 million resolved points ${ }^{6}$. On the other hand, a time domain approach employing a differential pulse-width pair (DPP) BOTDA system combined with optical pulse coding has led to $25 \mathrm{~cm}$ spatial resolution over a $60 \mathrm{~km}$ range, representing $250 \mathrm{k}$ independent points 7 .

Most of the time-domain approaches make use of a double-sideband (DSB) probe scheme to avoid depletion of the pump pulses; this is especially helpful in long-range Brillouin systems ${ }^{8,9}$. It has been recently found that schemes based on a DSB probe are substantially affected by spectral distortions of the pump pulse ${ }^{10}$, resulting from the conventional symmetrical scanning of the probe sidebands. Recently, a solution to this detrimental effect has been proposed ${ }^{11}$, in which the spectral separation between the two probe sidebands is kept constant, while the optical frequency of the pump is swept to scan the Brillouin spectral response. The technique has shown to be an efficient method to raise the probe power up to the threshold of the amplified spontaneous Brillouin scattering (ASpBS), enhancing the sensor performance.

In this paper we demonstrate that the strong Brillouin pump-probe interaction in a DPP-BOTDA sensor leads to huge distortion of the pump pulse. This detrimental effect is critical and nullifies the differential gain response required by the system, making it impossible to perform distributed sensing with tens of $\mathrm{cm}$ resolution over long distances. We here experimentally demonstrate that above-mentioned novel scanning scheme mitigates the distortion of the pulses, enabling distributed sensing with a sharp spatial resolution of $20 \mathrm{~cm}$ along a sensing distance of $37.5 \mathrm{~km}$. This represents $190 \mathrm{k}$ resolved points, obtained with an acquisition time of a few minutes.

*alejandro.dominguezl@uah.es

Sixth European Workshop on Optical Fibre Sensors, edited by Elfed Lewis, Proc. of SPIE Vol. 9916, 991635 - (c) 2016 SPIE · CCC code: 0277-786X/16/\$18 - doi: 10.1117/12.2236927 


\section{PRINCIPLES}

BOTDA systems are based on the interaction between two counter-propagating signals ${ }^{1,2}$ : one of them is a pulsed wave (pump) and the other one is a continuous-wave signal (probe wave). The probe signal is most-commonly generated by intensity modulation using a modulation frequency that sweeps around the Brillouin frequency shift (BFS) of the fiber. This modulation generates two probe sidebands, which had been proven to be an effective way to mitigate pump depletion and non-local effects when compared to single-sideband (SSB) modulation schemes ${ }^{8,9}$. However, it has been recently proven that DSB schemes also bring a spectral distortion on the pump pulse, inducing severe deformations in the Brillouin gain and loss spectra, and ultimately, perturbing the BFS determination ${ }^{10}$. Such a distortion arises when scanning the pump-probe frequency offset in a symmetrical way, where the gain and loss processes generated over the pump frequency (originated by the anti-Stokes and Stokes probe components respectively), lead to a non-zero net gain affecting the pump. This non-flat gain over the pump occurs when the pump-probe frequency offset is detuned from the average BFS $\left(v_{\mathrm{B}}\right)$ of the fiber. If the pump-probe frequency offset is set to properly match the average BFS, the gain and loss processes compensate, generating a flat gain over the pump, and thus, inducing no distortion on $\mathrm{it}^{11}$. It is worth mentioning that any spectral distortion induced on the pump pulse also leads to an equivalent temporal distortion, which dramatically impairs the spatial resolution of the system. It should be noted that this phenomenon scales with the probe power, hence, limiting the performance of BOTDA sensors.

In order to avoid any pump pulse distortion, a novel scanning method has been recently proposed ${ }^{11}$, which is depicted in Fig. 1. For this, the probe-wave modulation frequency is fixed, matching the dominating $v_{\mathrm{B}}$ of the fiber. This way, a perfect overlapping of the gain/loss processes generated by the anti-Stokes/Stokes components of the probe wave is ensured, leading to a flat net gain over the pump, and consequently, inducing no distortion on it. In this case, the pump pulse frequency is swept in order to properly scan the Brillouin gain/loss process.

In order to achieve sub-meter spatial resolution, the DPP technique is widely used ${ }^{5}$. Such a method is based on the subtraction of two

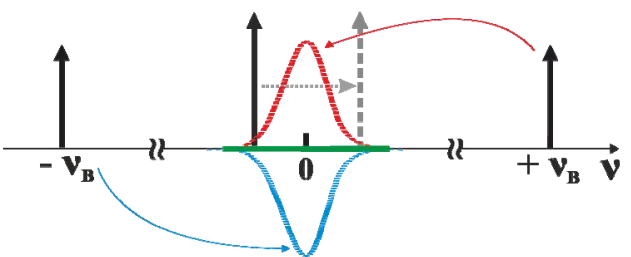

Fig. 1: Illustration of the proposed scanning technique, where the probe wave remains frequencyfixed at $v_{\mathrm{B}}$, inducing no distortion over the pump pulse. The pump frequency is swept to properly scan Brillouin gain and loss curves.

temporal traces, each of them obtained from the pump-probe interaction for different pulse widths. The system spatial resolution is determined by the width difference between the two pulses. For that reason, it turns critical to ensure wellshaped, non-distorted pulses. By combining DPP along with the above-described scanning technique ${ }^{11}$, the pulse distortion can be significantly mitigated, while allowing rising the probe power launched into the fiber. These features permit maintaining a considerably high spatial resolution over a long-range BOTDA sensor.

\section{EXPERIMENTAL RESULTS}

In order to support the above explained analysis we implement a DPP-BOTDA system, as shown in Fig. 2, in which the probe sidebands remain frequency fixed while the pump pulse scans the Brillouin gain spectrum. First, the laser beam is DSB-modulated by means of an intensity electro-optic modulator (EOM1), whose frequency is set to match the average BFS of the last section of the fiber (equivalent to the non-linear effective length $L_{\text {eff }}$ ). Afterwards, the modulated $\mathrm{CW}$ is split into two branches; one already constitutes the probe wave and is launched into the fiber-under-test (FUT) after going through a polarization switch (PS), which reduces polarization fading in the measured temporal traces. The other branch is used to generate the pump pulse. For this purpose, it is first necessary to select either the Stokes (Brillouin gain) or anti-Stokes (Brillouin loss) modulation sidebands by means of a suitable filter. In this case, a fiber Bragg grating (FBG) of $\sim 40 \mathrm{pm}$ of bandwidth is used to select the Stokes band, which is intensity-modulated by means of a second modulator (EOM2) at a frequency difference sweeping around the BFS $\left(v_{\mathrm{B}} \pm \Delta v\right)$. This new frequency component, which provides the pump wave, is selected through a narrow tunable filter ( $\sim 80 \mathrm{pm}$ of bandwidth), wide enough to allow a frequency tuning along the selected spectral span and narrow enough to filter out all unwanted spectral components. Subsequently, the pulse is properly shaped by means of an intensity modulator (EOM3), and then amplified by an Erbium doped fiber amplifier (EDFA). Then, after selecting the Brillouin gain component by means of another FBG, the signal is fed to a photo-receiver. In this case, a photoreceiver showing high transimpedance and a bandwidth of 350 $\mathrm{MHz}$; this bandwidth is high enough to detect changes in the signal according to the intended spatial resolution of $20 \mathrm{~cm}$. This setup is a slight modification of the BOTDA scheme implemented in Ref. 11. 


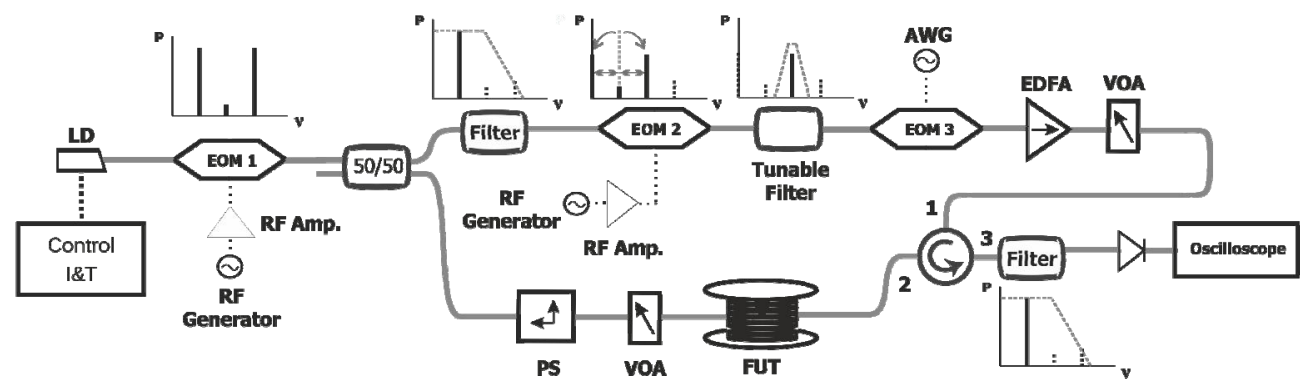

Fig. 2: Experimental setup. LD: Laser Diode; EOM: Electro-Optical Modulator; AWG: Arbitrary Waveform Generator; EDFA: Erbium Doped Fiber Amplifier; VOA: Variable Optical Attenuator; PS: Polarization Switch; FUT: Fiber Under Test.

In order to analyze the results obtained using the implemented scheme, measurements have been carried out over $37.5 \mathrm{~km}$ of SMF, presenting a considerably uniform BFS of $\sim 10.747 \mathrm{GHz}$ along the entire fiber. To perform DPP measurements, sharp squared pulses (200 ps rise/fall time) of $35 \mathrm{~ns}$ and $33 \mathrm{~ns}$ have been used, thus, resulting in a spatial resolution of $20 \mathrm{~cm}$ ( $2 \mathrm{~ns}$ width difference). The pump pulse peak power employed is $\sim 100 \mathrm{~mW}$ while the probe wave is $\sim 2.8 \mathrm{~mW}$ per sideband. Fig. 3a shows the acquired pump pulses after going through the fiber and interacting with the probe wave through SBS, as well as the subtraction among them that enables the DPP technique, for the conventional scanning method. Measured shapes illustrate the huge temporal distortion that the pump pulse experience when detuning the pump-probe frequency offset by $25 \mathrm{MHz}$. Such huge distortion provokes a null value as subtraction between the long and the short pulse (red line in Fig. 3a), turning the DPP technique not applicable for this range and powers. However, as it can be seen in Fig. 3b, by making use of the novel scanning procedure, the obtained pump pulses maintain their original shapes along the fiber, regardless the probe power used and its frequency detuning. This ensures the correct functioning of the DPP technique, having a well-shaped squared differential pulse of $\sim 2 \mathrm{~ns}$.
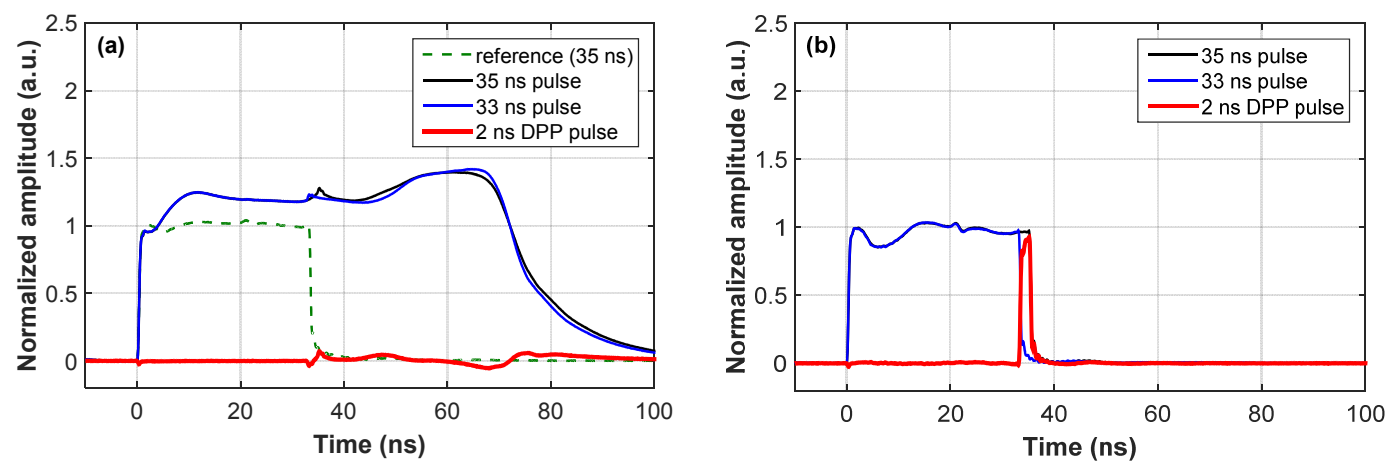

Fig. 3: Differential Pulse-Pair working principle, presenting a long pulse, a short one, and the resulting differential pulse. a) DPP on the conventional BOTDA sweeping method, showing a huge distortion in the pulses for a $25 \mathrm{MHz}$ pump-probe detuning (original non-distorted $35 \mathrm{~ns}$ pulse in green dashed-lines), and a null differential pulse. b) DPP on the novel scanning procedure: both pulses remain well-shaped, and its differential pulse shows a sharp squared shape and $2 \mathrm{~ns}$ width.

In order to verify the high spatial resolution provided by the system, a $20 \mathrm{~cm}$ section of a different fiber (presenting a different BFS) has been spliced near the end of a $37.5 \mathrm{~km}$ SMF spool. A full frequency sweep has been carried out, where all of the temporal traces have been averaged 2000 times, leading to a measuring time of a few minutes. Fig 4a shows the Brillouin gain spectrum (BGS) obtained at the very end of the fiber $(37.5 \mathrm{~km})$. The resulting spectrum presents no deformation and a width of $\sim 30 \mathrm{MHz}$ (measured as the full-width at half-maximum, FWHM), which further validates the proposed method. The conventional "bell shape" shown, as well as the sufficient signal-to-noise ratio (SNR) measured at the very end of the fiber $(4.3 \mathrm{~dB})$, make the system suitable to measure small BFS variations. The evolution of the BFS along the FUT has been determined, and a zoom in the last few meters of fiber is presented in Fig. 4b. The estimated BFS at each position demonstrates the presence of a slightly different piece of fiber, whose BFS is correctly determined to be $\sim 10.728 \mathrm{GHz}$. The frequency uncertainty measured at the end of the fiber is $\sim 1.9 \mathrm{MHz}$, which matches the expected theoretical value for the calculated 4.3 dB SNR. The spliced fiber is proven to be $20 \mathrm{~cm}$ long, which indeed verifies the system spatial resolution. It should be highlighted that such effective spatial resolution and sensing range corresponds to 190,000 sensing points, obtained in a conventional time-domain approach using a short acquisition time. 

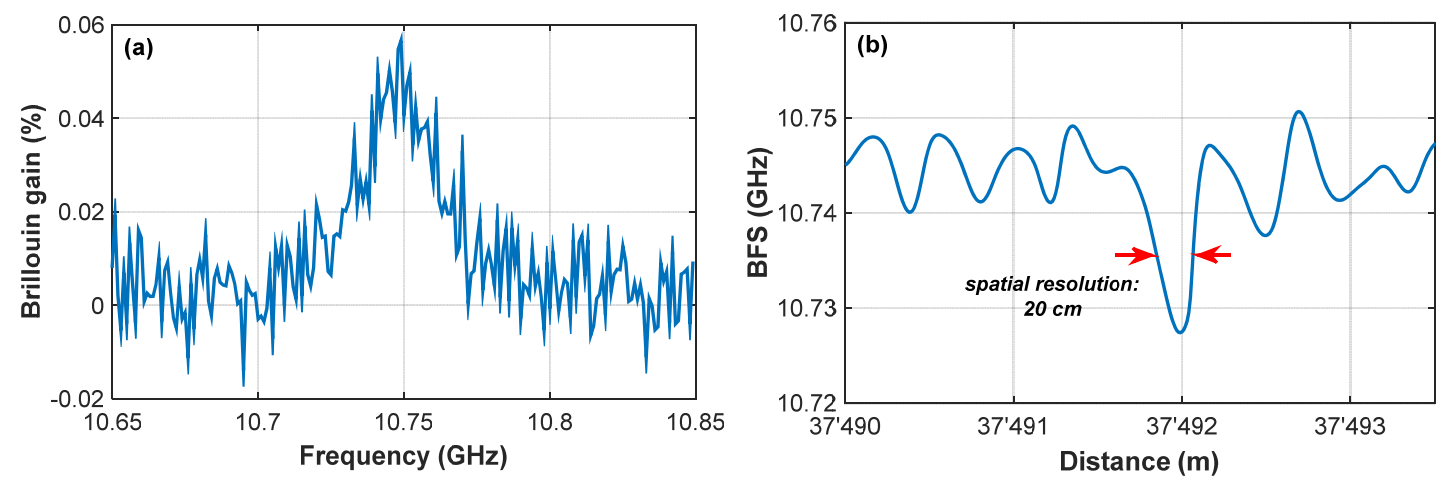

Fig. 3: a) BGS retrieved at the end of a $38 \mathrm{~km}$ SMF fiber for a DPP-BOTDA featuring $20 \mathrm{~cm}$ spatial resolution. B) BFS evolution at the far end of the fiber, where a $20 \mathrm{~cm}$ piece of different fiber was spliced.

\section{CONCLUSIONS}

In conclusion, an optimized long-range, high-resolution DPP-BOTDA sensor has been demonstrated. The use of the proposed novel scanning method ensures operating with non-distorted pump pulses (time and frequency wise) while allowing augmenting the probe wave power, ultimately limited by the onset ASpSBS threshold. Such power increase improves the overall system performance of the DPP-BOTDA technique, in this case allowing extending the sensing range up to $37.5 \mathrm{~km}$ featuring $20 \mathrm{~cm}$ spatial resolution. This results in achieving 190,000 sensing points along the fiber, which are measured in a few minutes, representing significant reduction of the acquisition time when compared to other methods, such as correlation-based approaches. In addition, the use of the proposed scanning procedure helps overcoming fundamental limits of high spatial resolution techniques, which had not been depicted until now, when applied to optimized BOTDA sensors. The potential of the present approach is high, as it could be also combined with other additional techniques in order to further improve the system performance.

This work was supported in part by the European Research Council through StG U-FINE (Grant 307441), in part by the Spanish MINECO under Project TEC2013-45265-R; and the Comunidad de Madrid under Project SINFOTON-CM:S2013/MIT-2790. The work of SML was supported by the Spanish MINECO through a "Ramón y Cajal" Contract.

\section{REFERENCES}

[1] Horiguchi, T., Shimizu, K., Kurashima, T., Tateda, M., Koyamada, Y., "Development of a distributed sensing technique using Brillouin scattering,” J. Lightw. Technol. 13(7), 1296-1302 (1995).

[2] Soto, M. A., Thévenaz, L., "Modeling and evaluating the performance of Brillouin distributed optical fiber sensors," Opt. Express 21, 31347-31366 (2013)

[3] Hotate, K. "Measurement of Brillouin gain spectrum distribution along an optical fiber using a correlation-based techniqueproposal, experiment and simulation," IEICE Trans. Electron. E83-C(3), 405-411 (2000).

[4] Zadok A, Antman Y, Primerov N, Denisov A, Sancho J, Thévenaz L, Random-access distributed fiber sensing, Laser \& Photon. Rev. 6, L1-L5 (2012).

[5] Li, W., et al., "Differential pulse-width pair BOTDA for high spatial resolution sensing," Opt. Express 16, 21616-21625 (2008).

[6] Denisov, A. Soto, M.A., Thévenaz, L., "Going beyond 1000000 resolved points in a Brillouin distributed fibre sensor: theoretical analysis and experimental demonstration" in Press Light: Science \& Applications (2016).

[7] Soto, M. A., Taki, M., Bolognini, G., and Di Pasquale, F., "Optimization of a DPP-BOTDA sensor with $25 \mathrm{~cm}$ spatial resolution over $60 \mathrm{~km}$ standard single-mode fiber using Simplex codes and optical pre-amplification” Opt. Express 20(7), 6860-6869 (2012)

[8] Thévenaz, L., Mafang, S. F., and Lin, J. "Effect of pulse depletion in a Brillouin optical time-domain analysis system" Opt. Express 21(12), 14017-14035 (2013).

[9] Minardo, A., Bernini, R., and Zeni, L., “A simple technique for reducing pump depletion in long-range distributed Brillouin fiber sensors,” IEEE Sens. J. 9(6), 633-634 (2009).

[10] Domínguez-López, A., Angulo-Vinuesa, X., López-Gil, A., Martín-López, S., and González-Herráez, M., "Non-local effects in dual-probe-sideband Brillouin optical time domain analysis” Opt. Express 23(8), 10341-10352 (2015).

[11] Domínguez-López, A., Yang, Z., Soto, M. A., Angulo-Vinuesa, X., Martín-López, S., Thévenaz, L., and González-Herráez, M., "Reaching the ultimate performance limit given by non-local effects in BOTDA sensors" Proc. SPIE 9634, 96342E (2015). 